\title{
LINEAR ASSIGNMENT PROBLEMS SOLVED BY GREEDY 2-OPT HEURISTICS ON GPU
}

\section{ROBERTO M. POVEDA CHAVES., ORLANDO GARCÍA HURTADO. \& EDWIN RIVAS TRUJILLO}

Engineering Faculty, Universidad Distrital Francisco José de Caldas, Bogotá, Colombia

\section{ABSTRACT}

We solve Linear Assignment Problems (LAP) through a Parallel Local Search Heuristics. LAP is properly represented as a Traveling Salesman Problem (TSP) which, in turn, is a particular case of Quadratic Assignment Problem (QAP). Our proposed method uses the Greedy 2-opt Local Search Heuristics proper to QAP and is fully implemented on Graphical Processing Units (GPUs).

KEYWORDS: Linear Assignment Problems (LAP), Traveling Salesman Problem (TSP), Quadratic Assignment Problem (QAP), Greedy 2-opt Local Search Heuristics, Graphics Processing Unit (GPUs)

Received: Aug 26, 2020; Accepted: Sep 16, 2020; Published: Nov 06, 2020; Paper Id.: IJMPERDOCT202028

\section{INTRODUCTION}

LAPs minimize the total cost to assign $m$ agents to $n$ tasks in the most efficient way, costs are represented by $c_{r s}$ and interpret the value to assign the agent $r$, to the task $s$ with $0 \leq r<n, 0 \leq s<m$, (Carter, 2018).

Assignation problems have been resolved using exact methods, the most usual solving technique is better known as the Hungarian method, (Taha, 2017). Otherwise, approximate methods, such as: Metaheuristics (Particle Swarm Optimization (PSO), Evolutionary Algorithms (EA) among others) and Heuristics (Local Search Methods, Greedy Randomized Adaptive Search among others) have been used in recent years for the solution of NP-hard combinatorial problems such as the TSP, (Dorigo, 1997)

Linear Arrangement Problem (The suitesparse matrix. The Harn Museum, 2011), Maximum Clique Problem (MCP) (School of Computing Science, 2012) and QAP (Gambardella, 1999). GPUs runs these approximate methods in parallel diminishing significantly execution time, (J, 2014).

\section{PRELIMINARIES}

\subsection{Linear Assignment Problem (LAP).}

The mathematical model of this problem is:

Minimize $Z=\sum_{r=0}^{m-1} \sum_{s=0}^{n-1} c_{r s} x_{r s}$

Subject to

$$
\sum_{s=0}^{n-1} x_{0 s}=1
$$




$$
\begin{gathered}
\sum_{s=0}^{n-1} x_{(m-1) s}=1 \\
\sum_{r=0}^{m-1} x_{r 0} \leq 1 \\
\vdots \\
\sum_{r=0}^{m-1} x_{r(n-1)} \leq 1
\end{gathered}
$$

where:

$$
x_{r s}=\left\{\begin{array}{c}
1, \text { if agent } r \text { is assigned to task } s \\
0, \text { otherwise }
\end{array}\right.
$$

A LAP is a special case of a transportation problem with agents $\left(A_{r_{s}}\right)$ as origins and tasks $\left(T_{s^{\prime} s}\right)$ as final destinations considering demands and offers equal to 1 (figure 1).

1

1

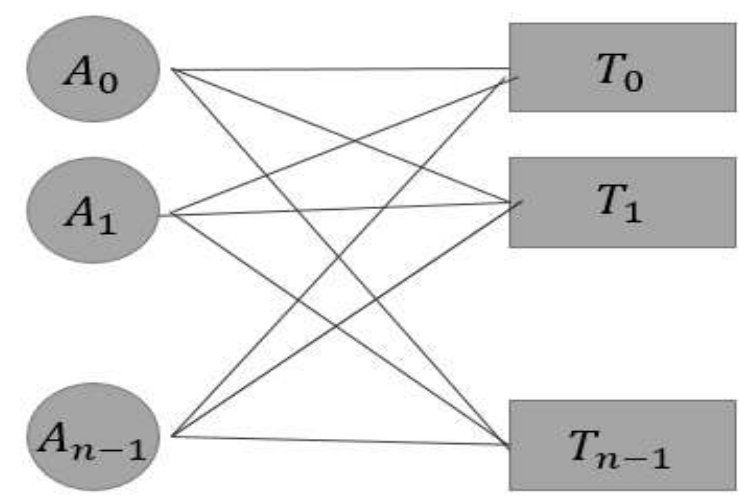

1

1

1

Figure 1: a LAP as a transportation problem

A LAP can be solved by considering the number of agents equal the number of tasks, creating fake agents or tasks with respective costs equal to zero. A method that solves this problem is the Hungarian method whose complexity is $O\left(n^{3}\right)$

\subsection{Traveling Salesman Problem (TSP).}

The closed TSP is a combinatorial problem of type NP-Complete that consists finding a Hamiltonian cycle of minimum length, in other words, a salesman visits to set of $n$ cities and returns to the starting city so that each city is just visited once and the cost of travel is minimum; if there is no return to the city of origin, the problem involves finding only a Hamiltonian path of length minimal (open TSP), i.e.

open TSP : $\min _{\pi \in Z_{n}} \sum_{r=0}^{n-1} d_{\pi(r) \pi(r+1)}$

where $D=\left(d_{r s}\right)$ is a distance matrix, and

$Z_{n}=\{\pi \mid \pi: N \rightarrow N\}$, where $N=\{0,1, \ldots, n-1\}$. 
2.3.Quadratic Assignment Problem (QAP). This problem tries to assign $n$ facilities to $n$ locations in such a way as to minimize both the flow between the facilities and the distance between the locations. The original mathematical model is (Loiola, 2007)

$$
\operatorname{Min}_{\pi \in Z_{n}} \sum_{r=0}^{n-1} \sum_{s=0}^{n-1} f_{r s} d_{\pi(r) \pi(s)}
$$

where $D=\left(d_{r s}\right)$ is a distance matrix, $F=\left(f_{r s}\right)$ is a flow matrix, $Z_{n}=\{\pi \mid \pi: N \rightarrow N\}$, where $N=\{0,1, \ldots, n-$ $1\}$.

The following formulation is equivalent to the previous formulation and is known as a trace formulation:

$$
\min _{X \in X_{n}} \operatorname{trace}\left(F X D^{t} X^{t}\right)
$$

where $X=\left(x_{r s}\right) ; X$ and $\pi$ are related like this:

$$
x_{r s}=\left\{\begin{array}{l}
1, \text { if } \pi(r)=s \\
0, \text { otherwise }
\end{array}\right.
$$

and $X_{n}$ is known as the set of matrices of permutations.

\subsection{Greedy 2-opt Local Search Heuristics.}

The Greedy 2-opt local optimization heuristics (Ji \& Wu, 2006), this method orderly swaps all two-by-two installations on the QAP locations, but as a better solution is found $\left(\Delta_{r s}<0\right.$ in the following formulation) it immediately updates along with its cost

$$
\begin{gathered}
\Delta_{r s}=\left(f_{r s}-f_{s r}\right)\left(d_{\pi(r) \pi(s)}-d_{\pi(s) \pi(r)}\right)+\left(F_{r .}-F_{s .}\right) \cdot\left(\left(D X^{t}\right)_{\pi(s) \cdot}-\left(D X^{t}\right)_{\pi(r)}\right) \\
+\left(F_{\cdot r}-F_{\cdot s}\right) \cdot\left((X D)_{\cdot \pi(s) \cdot}-(X D)_{\cdot \pi(r)}\right)
\end{gathered}
$$

here, $r, s$ are facilities that are exchanged, $f_{r s}=f_{s r}=0$ in the last addends. $F_{k}$. indicates the row $k$ of the matrix $F$ and $F_{.}$indicates the column $k$ of the matrix $F$.

The number of swaps performed in each iteration is exactly $\frac{n(n-1)}{2}$.

\subsection{Graphics Processing Unit (GPUs).}

These are devices that process images with great power of floating point operations, which frees up the workload of the CPU. Its most frequent use are the video games, but in 2006 the NVIDIA company of graphics processors designed the first GPU to execute programs written in CUDA $\mathrm{C} / \mathrm{C}++$ language programming model, taking advantage of its accelerated calculation. CUDA is a relevant architecture of parallel programming with a Single Instruction Multiple Data (SIMD) approach, giving rise to which is known as General-Purpose GPU computing (GPGPU) (Farber, 2012)(CUDA. Nvidia cuda programming guide, 2016).

\section{METHODOLOGY AND IMPLEMENTATION}

A LAP of size $n$ can be represented as an open TSP of size $2 n$ with nodes $A_{0}, A_{1}, \ldots, A_{n-1}, T_{0}, T_{1}, \ldots, T_{n-1}$ (see figure 1). The costs of the edges $\left(A_{r}, T_{s}\right)$ are the values $c_{r s}$, for $0 \leq r<n, 0 \leq s<n$. The costs of edges in the opposite direction are 0 . A permutation of the TSP has the form: 


$$
\pi=\llbracket 0, p(0), 1, p(1), 2, p(2), \ldots, n-1, p(n-1) \rrbracket
$$

In $\pi$ its components in the even positions $0,1,2, \ldots, n-1$ do not change from one iteration of another in our algorithm (represent agents) and the values in the odd positions of $\pi, p(0), p(1), p(2), \ldots, p(n-1)$ are a permutation of the set $n, n+1, n+2, \ldots, 2 n-1$ (represent tasks). if, in the QAP formulation, $D=\left(d_{r s}\right)$ is defined as the matrix of distances between cities of the TSP, and $F=\left(f_{r s}\right)$ is defined as a $n$ vertices hamiltonian path adjacency matrix, for example:

$$
F=\left(\begin{array}{ccccc}
0 & 1 & 0 & \cdots & 0 \\
0 & 0 & 1 & \cdots & 0 \\
\vdots & \vdots & \vdots & \ddots & \vdots \\
0 & 0 & 0 & 0 & 1 \\
1 & 0 & 0 & \cdots & 0
\end{array}\right)
$$

then:

open TSP : $\min _{\cdot \pi \in Z_{n}} \sum_{r=0}^{n-1} d_{\cdot \pi(r) \cdot \pi(r+1)} \leftrightarrow \min _{\cdot \pi \in Z_{n}} \sum_{r=0}^{n-1} f_{r(r+1)} d_{\cdot \pi(r) \cdot \pi(r+1)} \leftrightarrow \operatorname{Min}_{\cdot \pi \in Z_{n}} \sum_{r=0}^{n-1} \sum_{s=0}^{n-1} f_{r j} d_{\cdot \pi(r) \cdot \pi(s)}:$ QAP then, we finally solve the LAP as a QAP with initial $\pi$ permutation, distance matrix $\mathrm{D}=\left(d_{r s}\right)_{2 n \times 2 n}$.

$$
D=\left(\begin{array}{ll}
\mathbf{0} & C \\
\mathbf{0} & \mathbf{0}
\end{array}\right)
$$

where $\mathrm{C}=\left(c_{r s}\right)_{n \times n}$, and $\mathbf{0}$ is a matrix of zeros of size $n \times n$ and flow matrix $\mathrm{F}=\left(f_{r s}\right)_{2 n \times 2 n}$ an adjacency matrix of any hamiltonian path.

Each GPU block is used to represent the permutation $\pi$. Each GPU thread corresponds to a component of $\pi$. The Flow and Distance matrices reside in GPU constant memory to speed up calculations. We evaluated the cost of this permutation using trace formulation of QAP, since a GPU is a vector device (Cook, 2013).

For testing purposes, a custom CUDA program was written, the algorithms was executed in an IntelCoreTM i7 and GPU Nvidia GeForce GTX 760M. Details of this implementation can be consulted in (Poveda \& Gómez, 2018).

\section{CONCLUSIONS AND FUTURE WORK}

Linear Assignment Problems are solved in practice using exact algorithms such as the Munkres Faires's algorithm. This document proposes an alternative solution using QAP proprietary local search heuristic fully implemented on a GPU. The Linear Assignment Problem is posed as a transportation problem which in turn is a particular case of the TSP. The TSP is posed as a QAP equivalent and its stated objective function is optimized in a matrix form, ideal to be implemented in GPU.

Future works to get solutions more quickly could be to implement the objective function of the TSP (without using the QAP) in a matrix form and therefore consider a local search heuristic of this problem. It would take advantage of TSP's heuristics that have an efficient geometric sense to improve solutions (Lin Kernighan's heuristics) instead of the heuristic used in this document that proceeds exhaustively to improve solutions.

\section{REFERENCES}

1. Carter, M. (2018). Operations Research: A Practical Introduction. CRC Press.

2. Cook, S. (2013). CUDA Programming: A Developer's Guide to Parallel Computing with GPUs (Applications of Gpu Computing). Morgan Kaufmann. 
3. CUDA. Nvidia cuda programming guide. (2016). Obtenido de https://developer.nvidia.com/cuda-

4. Dorigo, M. (1997). Ant colonies for the travelling salesman problem. Biosystem, Elsevier, 73-81.

5. Farber, R. (2012). CUDA Application Design and Development. MK Nvidia.

6. Gambardella, L. (1999). nt colonies for the quadratic assignment problem. Journal of the Operational Research Society, 1678176.

7. J, C. (2014). Professional CUDA C Programming. Wiley.

8. Ji, P., \& Wu, Y. (2006). A Solution Method for the Quadratic. The Sixth International Symposium on Operations Research and Its Applications (ISORA'06), 106-117.

9. Loiola, E. (2007). A survey for the quadratic assignment problem. European Journal of Operational Research, 657-690.

10. Poveda, R., \& Gómez, J. (2018). Solving the quadratic assignment problem (qap) through a fime-grained parallel genetic algorithm implemented on gpus. In Springer Nature Switzerland AG (N. T. Nguyen et al. (Eds.): ICCCI 2018., ed.), LNAI 11056, (págs. 145-154). Bristol, England.

11. School of Computing Science. (2012).

12. Taha, A. (2017). Operations Research: An Introduction. Pearson.

13. The suitesparse matrix. The Harn Museum. (2011). Obtenido de The suitesparse matrix. The Harn Museum: http://www.cise.ufl.edu/research/sparse/matrices/ 

